\title{
Architecture of Dispatched, a Transmembrane Protein Responsible for Hedgehog Release
}

\author{
Yitian Luo ${ }^{1,2+}$, Guoyue Wan $^{1+}$, Xuan Zhou ${ }^{1+}$, Qiuwen Wang ${ }^{1,2}$, Yunbin Zhang ${ }^{1}$, Juan Bao ${ }^{1}$, \\ Yao Cong ${ }^{1}$, Yun Zhao ${ }^{3 *}$ and Dianfan $\mathrm{Li}^{1 *}$ \\ ${ }^{1}$ CAS Center for Excellence in Molecular Cell Science, Shanghai Institute of Biochemistry and Cell Biology, University of Chinese \\ Academy of Sciences, Chinese Academy of Sciences, Shanghai, China, ${ }^{2}$ School of Life Science and Technology, ShanghaiTech \\ University, Shanghai, China, ${ }^{3}$ School of Life Science, Hangzhou Institute for Advanced Study, University of Chinese Academy of \\ Sciences, Hangzhou, China
}

\section{OPEN ACCESS}

Edited by: Yasuteru Shigeta, University of Tsukuba, Japan

Reviewed by: Jennifer Cash, University of California, Davis, United States James Gerald Bann, Wichita State University, United States

*Correspondence: Yun Zhao yunzhao@sibcb.ac.cn Dianfan Li

dianfan.li@sibcb.ac.cn

${ }^{+}$These authors have contributed equally to this work

Specialty section:

This article was submitted to Molecular Recognition,

a section of the journal

Frontiers in Molecular Biosciences

Received: 06 May 2021 Accepted: 11 August 2021 Published: 07 September 2021

Citation:

Luo Y, Wan G, Zhou X, Wang Q, Zhang $Y$, Bao J, Cong $Y$, Zhao $Y$ and Li D (2021) Architecture of Dispatched, a Transmembrane Protein Responsible for Hedgehog Release.

Front. Mol. Biosci. 8:701826.

doi: $10.3389 /$ fmolb.2021.701826
The evolutionarily conserved Hedgehog $(\mathrm{Hh})$ signaling pathway is crucial for programmed cell differentiation and proliferation. Dispatched (Disp) is a 12-transmembrane protein that plays a critical role in the Hedgehog $(\mathrm{Hh})$ signaling pathway by releasing the dually lipidated ligand $\mathrm{HhN}$ from the membrane, a prerequisite step to the downstream signaling cascade. In this study, we focus on the Disp from water bear, a primitive animal known as the most indestructible on Earth. Using a zebrafish model, we show that the water bear homolog possesses the function of Disp. We have solved its structure to a 6.5- $\AA$ resolution using single-particle cryogenic electron microscopy. Consistent with the evolutional conservation of the pathway, the water bear Disp structure is overall similar to the previously reported structures of the fruit fly and human homologs. Although not revealing much detail at this resolution, the water bear Disp shows a different conformation compared to published structures, suggesting that they represent different functional snapshots.

Keywords: cryo-EM, hedgehog release, hedgehog signaling, membrane protein, three-dimensional structure

\section{INTRODUCTION}

Multi-cellular organisms rely on proper cell-cell communications mediated by controlled signaling transduction throughout the life cycle. The Hedgehog ( $\mathrm{Hh}$ ) signaling pathway is essential in embryonic morphogenesis in metazoans (Niewiadomski et al., 2019). The malfunction of the pathway results in the clustering of "hairy" denticles in Drosophila (fruit fly) larvae, a phenotype that inspired the name of the hedgehog gene (Nüsslein-Volhard and Wieschaus, 1980). In humans, abnormal Hh activities are associated with congenital craniofacial deformities (Nieuwenhuis and Hui, 2005) and tumorigenesis (Otsuka et al., 2015; Niewiadomski et al., 2019). In the absence of signals, the pathway is suppressed by a multi-crossing transporter named Patched (Ptc) which depletes sterol in the inner leaflet of the plasma membrane (Myers et al., 2017; Zhang et al., 2018). The Hh ligand released by producing cells binds to Ptc, inhibiting the sterol-transport function and activating the downstream signaling cascade that leads to the transcription of several genes in the pathway (Hui and Angers, 2011).

$\mathrm{Hh}$, the ligand of the pathway, is a family of small proteins sharing similar features. Among them, the Sonic Hh (Shh) is the most studied. After its synthesis in the producing cells, the pre-protein is first cleaved by its own C-terminal catalytic domain. A cholesterol molecule is then attached to the 
newly exposed C-terminus of the $\mathrm{N}$-terminal half $(\mathrm{HhN})$ (Hall and Cleverdon, 2019), followed by a second lipidation step at its $\mathrm{N}$-terminus by the Hh acyltransferase (Pepinsky et al., 1998). The dual lipidation makes the mature $\mathrm{HhN}$ ligands hydrophobic and may serve as a "barcode" for its intracellular trafficking from the endoplasmic reticulum or Golgi to the plasma membrane where $\mathrm{Hh}$ is anchored by the lipid moieties (Mao et al., 2009; Kornberg, 2011; Simon et al., 2016). To reach and affect receiving cells in long-range signaling events such as tissue patterning (Etheridge et al., 2010), Hh must be firstly released from the membrane of the producing cells. This process is facilitated by the transmembrane protein Dispatched (Disp) (Burke et al., 1999). Consistent with this important role, malfunction of Disp dispatterns $\mathrm{Hh}$ concentration gradient and causes developmental abnormalities in long bones in mice embryo (Tsiairis and McMahon, 2008).

Disp $(\sim 120 \mathrm{kDa})$ belongs to the resistance-nodulationdivision (RND) family (Nikaido, 2018), members of which include the aforementioned Hh receptor Ptc (Qi et al., 2018a; Qi et al., 2018b; Gong et al., 2018; Zhang et al., 2018; Qi et al., 2019; Qian et al., 2019; Rudolf et al., 2019; Zhang et al., 2020; Luo et al., 2021); the cholesterol transporter NPC1 (Gong et al., 2016; Li et al., 2016; Long et al., 2020; Qian et al., 2020); and a variety of bacterial transporters such as AcrB (Seeger et al., 2006). Structural studies have revealed common features of RND transporters (Nikaido, 2018). They typically contain a membraneembedded domain splitting into two pseudosymmetrical halves with six transmembrane helices (TMHs) each and two extracellular domains that are associated with the two transmembrane halves. RND transporters are known to use electrochemical gradients such as proton- and sodium-motive force as the energy source to drive the conformational cycles (Pos, 2009; Myers et al., 2017). Consistently, three negatively charged residues in the transmembrane domain of Ptc (Asp499, Asp500, and Glu1081 in murine Ptc) are proposed to sense electrochemical gradient and to coordinate the conformational changes (Taipale et al., 2002; Myers et al., 2017; Zhang et al., 2018). Unlike Ptc, of which the transporter function is supported by structural and mutagenesis studies (Myers et al., 2017; Zhang et al., 2018), evidence for Disp being a transporter is somewhat elusive: the cholesterol moiety is required for Disp-mediated $\mathrm{Hh}$ release (Tukachinsky et al., 2012), and the genetic neutralization of the corresponding intramembrane negatively charged residues causes loss of function (Ma et al., 2002).

Recently, the structures of Disp from fruit fly (ffDisp) (Cannac et al., 2020) and human ( $h$ Disp) (Chen et al., 2020) were determined by two independent research groups using cryogenic electron microscopy (cryo-EM). The structural studies revealed its architecture with two similarly sized ECDs connected to the 12-TMH domain. In addition, both groups reported the low-resolution map of Disp in complex with the ligand $\mathrm{Hh}$, providing direct evidence for the interaction and revealing the interface information for the binding. Despite the evolutional distance, ffDisp and $h$ Disp share a superimposable architecture, thus likely representing the same functional conformation snapshot. Obtaining molecular images at different conformations will be helpful to understand its function mechanism. For membrane transporters and channels, this may be achieved by structural studies using conformation-locking mutants (Bozzi et al., 2016; Vuppada et al., 2018; Galochkina et al., 2019), by exploring different membrane environments such as lipid nanodiscs (Gao et al., 2016; Mi et al., 2017; Matthies et al., 2018; Kalienkova et al., 2019; Reddy et al., 2019; Luo et al., 2021), by screening conformationspecific antibodies (Zimmermann et al., 2018; Kumar et al., 2021), and by screening different experimental conditions including protein homologs (Newstead et al., 2011; Solcan et al., 2012; Doki et al., 2013) and buffer conditions (Chang et al., 2014; Qian et al., 2020). In terms of homologs, we had an interest in water bear proteins. Water bears are the most resilient animal known because of their ability (as a collective term for many species) to endure conditions such as exposure to extreme temperatures $\left(-272^{\circ} \mathrm{C}\right.$ and $\left.150^{\circ} \mathrm{C}\right)$, extreme pressures $(6,000$ atmospheres and vacuum), air deprivation, radiation, dehydration, and starvation (Sloan et al., 2017; Orellana et al., 2018). Despite the emerging interests in using water bear as model systems to study evolutional development (Gabriel et al., 2007; Goldstein, 2018), structural information for their proteins are scarce (Fukuda et al., 2017; Fukuda and Inoue, 2018; Kim et al., 2019) and no membrane protein structures of water bear origin have been reported.

In this study, we report the characterization, expression, purification, and cryo-EM structure determination of Disp from Hypsibius dujardini, a type of water bear. The water bear Disp ( $w b$ Disp) has an overall similar structure with ffDisp and $h$ Disp, despite that they only share modest sequence homology. Interestingly, the transmembrane domain of $w b$ Disp shows a slightly different conformation compared to the published structures. The observations raise the possibility of the structural differences being related to Disp's function and the structures provide a preliminary framework to design experiments to test this hypothesis.

\section{MATERIALS AND METHODS}

\section{Molecular Cloning}

The DNA encoding Disp from water bear (NCBI OQV19566.1) was obtained by PCR using overlapping oligos that are 60 to 90 -nt long. The resulting fragments were Gibson assembled into a pEG_BacMam-backbone vector named pBHGS (Goehring et al., 2014). The polypeptide sequence of protein expressed using this vector contains amino acids of, from $\mathrm{N}$ - to C-terminus, as follows: a $9 \times$ His tag, the protein of interest, a $3 \mathrm{C}$ protease site, a thermostable green fluorescent protein (Yao et al., 2020), and a twin-Strep tag. Deletions were made by standard PCR-based protocols. DNA sequences were verified by sequencing.

\section{Protein Expression and Purification}

Disp homologs were transiently expressed in Expi293 cells as follows. For small-scale expression, typically, $15 \mathrm{ml}$ of cells (> $95 \%$ viability) at a density of $2 \times 10^{6} \mathrm{ml}^{-1}$ was diluted by two folds and cultured for another $24 \mathrm{~h}$ at $37^{\circ} \mathrm{C}$ in a $100 \mathrm{ml}$ flask in a $\mathrm{CO}_{2}$ 
incubator to re-reach a cell density of $2 \times 10^{6} \mathrm{ml}^{-1}$. Cells were aliquoted to 6-well plates for small-scale expression tests. Plasmid $(5 \mu \mathrm{g})$ and polyethylenimine (Cat. 23,966-2, Polysciences, $10 \mu \mathrm{g}$ ) were first separately incubated with $200 \mu \mathrm{L}$ of 293 medium (Cat. UP0050, Union Biotech, China) at room temperature (RT, $18-22^{\circ} \mathrm{C}$ ) for $3 \mathrm{~min}$. The two mixtures were then combined and incubated at RT for $20 \mathrm{~min}$ before being added into $2 \mathrm{ml}$ of cell culture. Sodium valproate (Cat. P4543-100G, Sigma) was included as an additive (Wulhfard et al., 2010) to increase expression at a final concentration of $2 \mathrm{mM} 12 \mathrm{~h}$ after transfection. After $40 \mathrm{~h}$, cells were harvested by centrifugation, flash-frozen in liquid nitrogen, and stored at $-80^{\circ} \mathrm{C}$ until use. For large-scale expression, the same protocol was followed except that $0.5 \mathrm{~L}$ of cells was seeded to $1 \mathrm{~L}$ of culture and the transfection was performed with $2.5 \mathrm{mg}$ of plasmids premixed with $5 \mathrm{mg}$ of polyethylenimine.

For purification, biomass from $2 \mathrm{~L}$ of culture was re-suspended lysis buffer (0.5 mM EDTA, $20 \mathrm{mM}$ HEPES pH7.5 and $150 \mathrm{mM}$ $\mathrm{NaCl}$ ) supplemented with $2 \mathrm{mg} \mathrm{ml}^{-1}$ iodoacetamide, complete protease inhibitor cocktail (Cat. 11836153001, Roche). Solubilization was carried out by adding $1 \%(\mathrm{w} / \mathrm{v}) n$-dodecyl $\beta$-D-maltoside (DDM) plus $0.2 \%(\mathrm{w} / \mathrm{v})$ cholesteryl hemisuccinate (CHS) to the cells with mild agitation at $4^{\circ} \mathrm{C}$ for $20 \mathrm{~min}$. Cell debris was removed by centrifugation at $48,000 \mathrm{~g}$ for $1 \mathrm{~h}$. The supernatant was mixed with Strep Tactin resin (Cat. 2-1201-010, IBA) that had been pre-equilibrated with the Buffer A and mixed gently for $2 \mathrm{~h}$. After batch binding, the beads were loaded to a gravity column and washed by 15 column volume (CV) of $0.1 \%$ digitonin in Buffer A. $w b$ Disp-GFP was then eluted from the column by $5 \mathrm{mM}$ of dethiobiotin and $0.1 \%$ digitonin in Buffer A. The eluted $w b$ Disp-GFP fractions were quantified by absorbance at $493 \mathrm{~nm}\left(\varepsilon_{493}=63,973 \mathrm{M}^{-1} \mathrm{~cm}^{-1}\right)$ and the fluorescent tag was removed by $3 \mathrm{C}$ protease cleavage at $4^{\circ} \mathrm{C}$ overnight. The mixture was then centrifuged at $21,000 \mathrm{~g}$ for $10 \mathrm{~min}$ before being loaded onto a Superose 6 10/300 GL column pre-equilibrated by Buffer A containing $0.1 \%$ digitonin for gel filtration. Peak fractions were pooled and concentrated to $3 \mathrm{mg} \mathrm{ml}^{-1}$.

\section{Fluorescence-Detection Size Exclusion Chromatography (FSEC) Assay for Expression and Thermostability}

FSEC assays (Hattori et al., 2012; Yao et al., 2020) were carried out to assess expression level and apparent thermostability as follows. Cells expressing Disp homologs or variants with a thermostable GFP (TGP) tag were re-suspended in $200 \mu \mathrm{L}$ of FSEC Buffer (0.2 $\mathrm{mM}$ tris(2-carboxyethyl)phosphine (TCEP), $50 \mathrm{mM} \mathrm{NaCl}$, and $20 \mathrm{mM}$ Tris- $\mathrm{HCl} \mathrm{pH} 8.0,0.5 \mathrm{mM}$ EDTA) supplemented with $1 \times$ protease inhibitor cocktail. DDM and CHS were added to a final concentration of $1 \%(\mathrm{w} / \mathrm{v})$ of DDM and $0.2 \%(\mathrm{w} / \mathrm{v})$ of CHS for solubilization at $4^{\circ} \mathrm{C}$ for $20 \mathrm{~min}$. The solubilized fraction was clarification by centrifugation at $21,000 \mathrm{~g}$ for $30 \mathrm{~min}$ before FSEC assays below.

To assess their relative expression level, $2 \mu \mathrm{L}$ of solubilized materials was loaded onto a ZenixC-300 column in a Shimadzu or Agilent HPLC machine equipped with a fluorescence detector for signal monitoring at the $482 / 508 \mathrm{~nm}$ pair. The mobile phase contained $0.03 \% \mathrm{DDM}(\mathrm{w} / \mathrm{v})$ and $0.006 \%$ CHS (w/v), $0.2 \mathrm{mM}$ TCEP, $50 \mathrm{mM} \mathrm{NaCl}, 0.5 \mathrm{mM}$ EDTA, and $20 \mathrm{mM}$ Tris- $\mathrm{HCl} \mathrm{pH}$ 8.0. The intensity and profile of the FSEC peak at desired $\mathrm{V}_{\mathrm{e}}$ were compared between Disp homologs, Disp mutants, or expression conditions such as time post-transfection, temperature, and types (sodium valproate and sodium butyrate) and concentration of additives.

For FSEC-based thermostability assays, $50 \mu \mathrm{L}$ of cell lysate was heated in a PCR machine for $20 \mathrm{~min}$, followed by $4^{\circ} \mathrm{C}$ incubation for $10 \mathrm{~min}$. The heated samples were clarified by centrifugation before being loaded onto a ZenixC-300 column in a Shimadzu or Agilent HPLC machine equipped with a fluorescence detector for signal monitoring at the $482 / 508 \mathrm{~nm}$ pair. The $\%$ fluorescence of the DispTGP peak was plotted against temperature and the apparent melting temperature $\left(T_{\mathrm{m}}\right)$ was obtained by regression fitting.

\section{Zebrafish Rescue Assay}

Zebrafish (Danio rerio) of the $\mathrm{AB}$ strain was provided by the Zebrafish Core Facility at the authors' institute, and all experimental protocols were approved by the Institutional Animal Care and Use Committee. sgRNAs were designed using the CRISPR Design webserver CCTop (https://crispr.cos. uni-heidelberg.de/index.html) and/or CHOPCHOP (http:// chopchop.cbu.uib.no/). DNA templates for sgRNAs were PCRamplified using the following forward primers containing a T7 promoter (italic) and guide sequence (Disp11, 5'- TAATACGAC TCACTATAGGTGTCCCAGCATTCAGGACCGGTTTTAGA GCTAGAAATAGC-3'; Disp12, 5’- TAATACGACTCACTA TAGGATGGGATCACGACTATAAGTTTTAGAGCTAGAAA TAGC-3'; Disp13, 5' - TAATACGACTCACTATAGGAGTTGAA GACCACTCGATATGTTTTAGAGCTAGAAATAGC-3') and the reverse primer with the standard chimeric sgRNA scaffold (ScaRev, 5'-AAAAGCACCGACTCGGTGCCACTTTTTCAA GTTGATAACGGACTAGCCTTATTTTAACTTGCTATTTCT AGCTCTAAAAC-3') (Varshney et al., 2015). sgRNAs commentary to the PCR products was synthesized in vitro using the HiScribe T7 Quick High Yield RNA Synthesis Kit (New England Biolabs, Cat. E2050S). To generate DNA temperate for the mRNA of $w b$ Disp, PCR was performed with the primer pair $w b \mathrm{D}$-Fwd (5'- TAATACGACTCACTATAGGA TGCCGAGACAACTCGTGCGACGGC-3') and $w b$ D-Rev (5'TCACTCGGTCGTCCGTCGCACCC-3') using the pBHGS$w b$ Disp plasmid as the template (in the primer sequence, italic denotes sequence representing a T7 promoter). In vitro synthesis of mRNA containing a 5'-cap and a 3'-poly(A) tail was performed using the mMessage mMachine SP6 kit (Ambion, Cat. AM1340). Cas9 ribonucleoprotein (RNP) complexes were prepared with Cas9 protein and sgRNAs as previously described (Varshney et al., 2015). The RNPs were injected into one-cell stage zebrafish embryos with or without mRNAs. Each embryo was injected with $1 \mathrm{~nL}$ of a solution containing $5 \mu \mathrm{M}$ Cas 9 and $1 \mu \mathrm{g} \mathrm{L}{ }^{-1}$ sgRNA or $\sim 5 \mu \mathrm{M}$ Cas $9,1 \mu \mathrm{g} \mu \mathrm{L}^{-1}$ sgRNA, and $160 \mathrm{ng} \mu \mathrm{L}^{-1}$ mRNA. 24 hours after fertilization (hpf), larvae were fixed in $4 \%$ paraformaldehyde (PFA; w/v, $\mathrm{pH} 7.4$ ) overnight at $4^{\circ} \mathrm{C}$, washed with PBS, and fluorescently stained with 4,6-diamidino-2phenylindole (DAPI). All specimens were washed with PBS before imaging under an Olympus FV3000 confocal laser scanning microscopes. 


\section{Cryo-EM Sample Preparation and Data Acquisition}

Two microliters of protein was applied to a Quantifoil Cu R1.2/ 1.3 (200 mesh) grid that was glow-discharged followed by blotting using Vitrobot Mark IV (FEI company) and flash-vitrified in liquid ethane. Movie stacks were acquired using a 300-kV Titan Krios transmission electron microscope (Thermo Fisher) equipped with a Cs corrector onto a K2 Summit direct electron detector (Gatan) in super-resolution mode. The pixel size was 1.0 ̊ after 2 times of binning (Supplementary Table S1). Each movie was dose-fractioned into 38 frames using a dose rate of $8 \mathrm{e}^{-}$per physical pixel per second on the detector. The exposure time was $7.6 \mathrm{~s}$ ( $0.2 \mathrm{~s}$ for each frame) with a total dose of $60.8 \mathrm{e}^{-} / \AA^{2}$. Defocus values varied from -1.5 to $-2.5 \mu \mathrm{m}$. All the images were collected with the SerialEM automated data collection software package (Mastronarde, 2005).

\section{Cryo-EM Data Processing}

Data processing was carried out using modules either belonging to or available through Relion 3.0 and 3.1 (Supplementary Figure S2) (Scheres, 2012a; Scheres, 2012b). Two datasets containing 2,852 and 2,528 movie stacks were motion-corrected and dose-weighted before further processing. Parameters of the contrast transfer function (CTF) were estimated using Ctffind 4 (Mindell and Grigorieff, 2003) and micrographs with CTF fitting quality better than $4 \AA$ were used for particle extraction. To construct a template for automatic particle picking, we manually selected $\sim 2,000$ particles for reference-free 2D classification. A total of 433,048 particles from dataset one and 345,698 particles from dataset two were obtained after removing bad particles. The particles were twofold downsampled to accelerate processing and subjected to several rounds of 2D classification, resulting in 184,167 and 182,925 clean-up particles from each subset. Subsequently, 3D classification was performed on each set using particles from selected 2D classes. After adjusting parameters (class number $\mathrm{K}$, regularization parameter $\mathrm{T}$, and particle alignment parameters), $3 \mathrm{D}$ classes with clear structural features were selected from each dataset. This resulted in 84,596 particles in Dataset 1 and 69,183 particles in Dataset 2. Further rounds of $3 \mathrm{D}$ classification using combined particles from the two datasets yielded a class with 62,850 particles. These particles were refined with a protein-only mask and further applied to CTF refinement and Bayesian polishing. Polished particles were refined with the mask and sharpened into a $6.5 \AA$ map by post-processing. Although the second refinement step did not improve the overall resolution, it yielded a map displaying more features in TMHs. Local resolution was estimated using Relion (Scheres, 2012b).

\section{Model Building and Validation}

Model of the ffDisp structure (PDB ID 6tbu) trimmed to Ca was fit to the 6.5- $\AA$ map using UCSF Chimera (Pettersen et al., 2004). The model was then manually adjusted based on the cryo-EM density map using Coot (Emsley et al., 2010) and refined in real space using Phenix (Liebschner et al., 2019). The resulting model was finely adjusted to fix geometry violations guided by the cryoEM density map using Coot.

\section{RESULTS AND DISCUSSION}

\section{wbDisp Shows a Higher Expression Level Than Other Homologs}

To elucidate the then-unknown architecture of Disp, we first set to overexpress, purify, and determine the cryo-EM structure of the murine homolog ( $m$ Disp) as it is one of the most studied in the literature. $m$ Disp was expressed in Expi293 cells with a thermostable green fluorescence protein (TGP) fused to its C-terminal (Cai et al., 2020). This allows convenient assessment of gel filtration behavior and expression level without purification using fluorescencedetector size exclusion chromatography (FSEC). As shown in Figure 1A, multiple peaks were observed for $m$ Disp due to degradation and aggregation. The main peak had a $\mathrm{V}_{\mathrm{e}}$ of $2.24 \mathrm{ml}$ which was similar to the $\mathrm{V}_{\mathrm{e}}$ of the monomeric (Luo et al., 2021) Ptc1 (2.40 ml), a homolog of Disp with a similar architecture. Thus, $m$ Disp likely formed a monomer under current conditions.

Preliminary purification of $m$ Disp using Strep resin only yielded $\sim 50 \mu \mathrm{g}$ of protein with insufficient purity $(\sim 70 \%)$ from $1 \mathrm{~L}$ of $m$ Disp-overexpressing mammalian cells. This relatively low yield prompted us to screen homologs for higher expression levels by FSEC analysis (Cai et al., 2020). Thus, six additional homologs were expressed in mammal cells on small scales, and the relative yields reflected by the peak intensities were assessed by FSEC using detergent-solubilized lysates. Among seven homologs (water bear, sea urchin, coral, slug, scorpion, pupfish, and mouse) (Supplementary Figure S2), wbDisp showed about 20 -fold higher expression than $m$ Disp. When superposed, $w b$ Disp showed a similar FSEC profile to $m$ Disp (Figure 1A).

\section{wbDisp Partially Rescues Somite Structure in disp Knock-out Zebrafish}

To investigate the function of $w b$ Disp, a phenotype-rescue assay was performed as follows to see if $w b$ Disp mediates Hh signaling in zebrafish. We injected mRNA of $w b$ Dsip and $m$ Disp into the embryo of zebrafish where the endogenous disp was knocked out using CRISPR/Cas9. In the absence of disp, the somite structure of zebrafish transformed from a V-shape (Figure 1B) to a $\mathrm{U}$-shape (Figure 1C). Injecting mRNA of $m$ Disp into the embryo completely rescued the phenotype (Figure 1D) while injecting $w b$ Dsip mRNA resulted in partial rescue (Figure 1E), suggesting that $w b$ Disp can partially recover Disp's activity. It may be that the non-native lipid environment and non-native HhN ligand (sequence identity of 59.6\%) (Supplementary Figure S3) were not optimal for the heterogeneously expressed $w b$ Dsip, resulting in an incomplete rescue.

\section{Purification and Negative Staining of wbDisp}

To further optimize the expression level, we screened several $w b$ Disp constructs by removing regions that are predicted (McGuffin et al., 2000) to be flexible (Supplementary Figure S4). First, the C-terminus (residues 986-1192, inclusive) was 

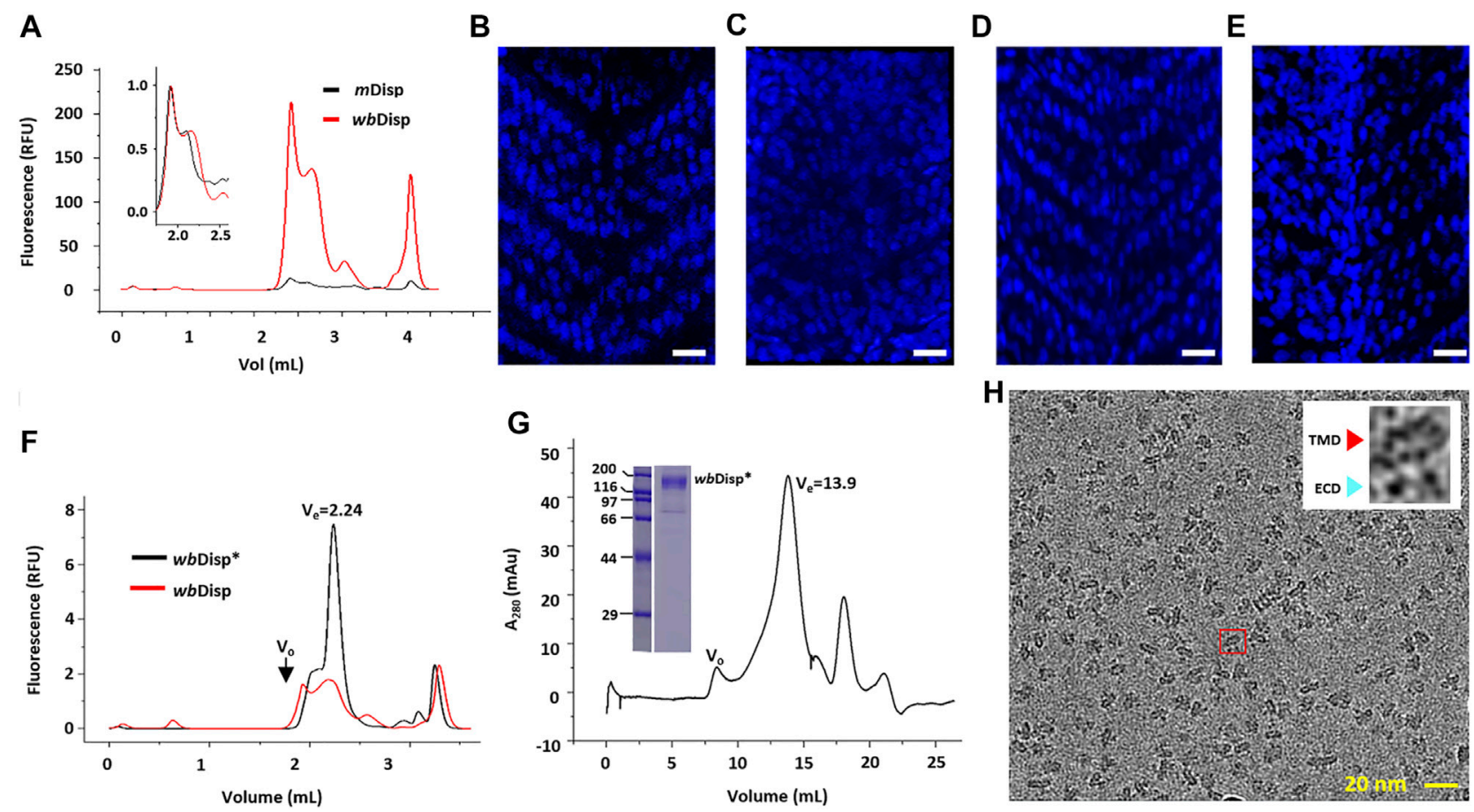

FIGURE 1 | Screening Disp homologs for structural and functional studies. (A) Fluorescence-detection size exclusion chromatography (FSEC) of Disp from mouse ( $m$ Disp) and water bear (wbDisp). The void volume was $1.80 \mathrm{ml}$ and the total volume was $4.20 \mathrm{ml}$. (B-E) Phenotype-rescue assay of wbDisp. The $\mathrm{V}$-shaped somites seen in zebrafish (B) were lost upon the knockout of the endogenous disp gene (C). This phenotype could be fully or partly rescued by introducing $m$ Disp (D) or wbDisp mRNA to the embryo (E). Bar $=40 \mu \mathrm{m}$. The brightness and contrast of (C) were adjusted as a whole for better contrast. (F) FSEC of the wild-type wbDisp and wbDisp* which contained several deletions (see text). (G) Preparative gel filtration of wbDisp. The void volume (V) is appropriately labeled. Inset shows the SDS-PAGE of a main-peak fraction. Molecular standards for electrophoresis are shown as kDa on the left. (H) A typical cryo-EM micrograph of wbDisp purified in digitonin. Inset shows the expanded image of a particle (red box) with a typical side view displaying a transmembrane domain (TMD) and two extracellular domains (ECDs).

systematically truncated by $50-70$ residues at a time. The construct containing residues 1-1116 was further screened by deletion at the internal loops. Specifically, the regions spanning residues 80-160, 530-550, and 660-680 were firstly systematically tested by removing approximately ten residues at a time. The ones with higher yields were then combined. This yielded the construct (dubbed $w b$ Disp $^{*}$ ) that consists of residues (1-111, 123-530, 542-1116) (Supplementary Figure S4). The optimized construct showed a three-fold higher expression level compared to the wild type (Figure 1F). To assess its stability, we performed the FSEC-thermostability assay (Hattori et al., 2012; Cai et al., 2020) of wbDisp using the tagged TGP as the reporter. Thus, the samples were heated for $20 \mathrm{~min}$ under different temperatures and the fluorescence intensity of the FSEC peak was plotted against temperature. As shown in Supplementary Figure S5A, $w b$ Disp $^{\star}$ displayed an apparent $T_{\mathrm{m}}$ of $79^{\circ} \mathrm{C}$. Compared to other membrane proteins (Cai et al., 2020), the denaturation profile shows a relatively slow decrease in the descending phase. This may indicate reversible denaturation.

To purify $w b$ Disp, the protein was solubilized in DDM/ CHS (1\% DDM and $0.2 \%$ CHS) and the detergent was exchanged to $0.1 \%$ digitonin on a Strep-Tactin column.
On gel filtration, $w b \mathrm{Disp}^{*}$ showed a reasonably symmetrical main peak. When analyzed by SDS-PAGE, the peak fraction contained mainly the target protein (Figure 1G). Negative-stain of purified $w b \mathrm{Disp}^{*}$ showed well-dispersed particles with views consistent with a membrane protein with two extracellular loops (Supplementary Figure S5B).

\section{Cryo-EM Structure of wbDisp*}

To determine its $3 \mathrm{D}$ structure, $w b \mathrm{Disp}^{\star}$ purified in digitonin was subjected to cryo-EM analysis. A $6.5-\AA$ map was reconstructed using 62,850 particles (Figure 1H, Figures 2A-E, Supplementary Figure S6-S8), showing a slingshot shape with the two extracellular blobs as the branches and the transmembrane helices as the handle (Figure 2B). To obtain a model at this low resolution, the recently published structure of the fruit fly homolog (ffDisp) (Cannac et al., 2020) was trimmed to poly-alanine and fit into the map, followed by manual mutation and adjustments.

WBDisp showed a monomeric structure with 12 transmembrane helices (Figures 2F,G). In literature, trimeric assembly by C-terminal regions has been reported for the dog Disp (Etheridge et al., 2010). Because our constructs were 


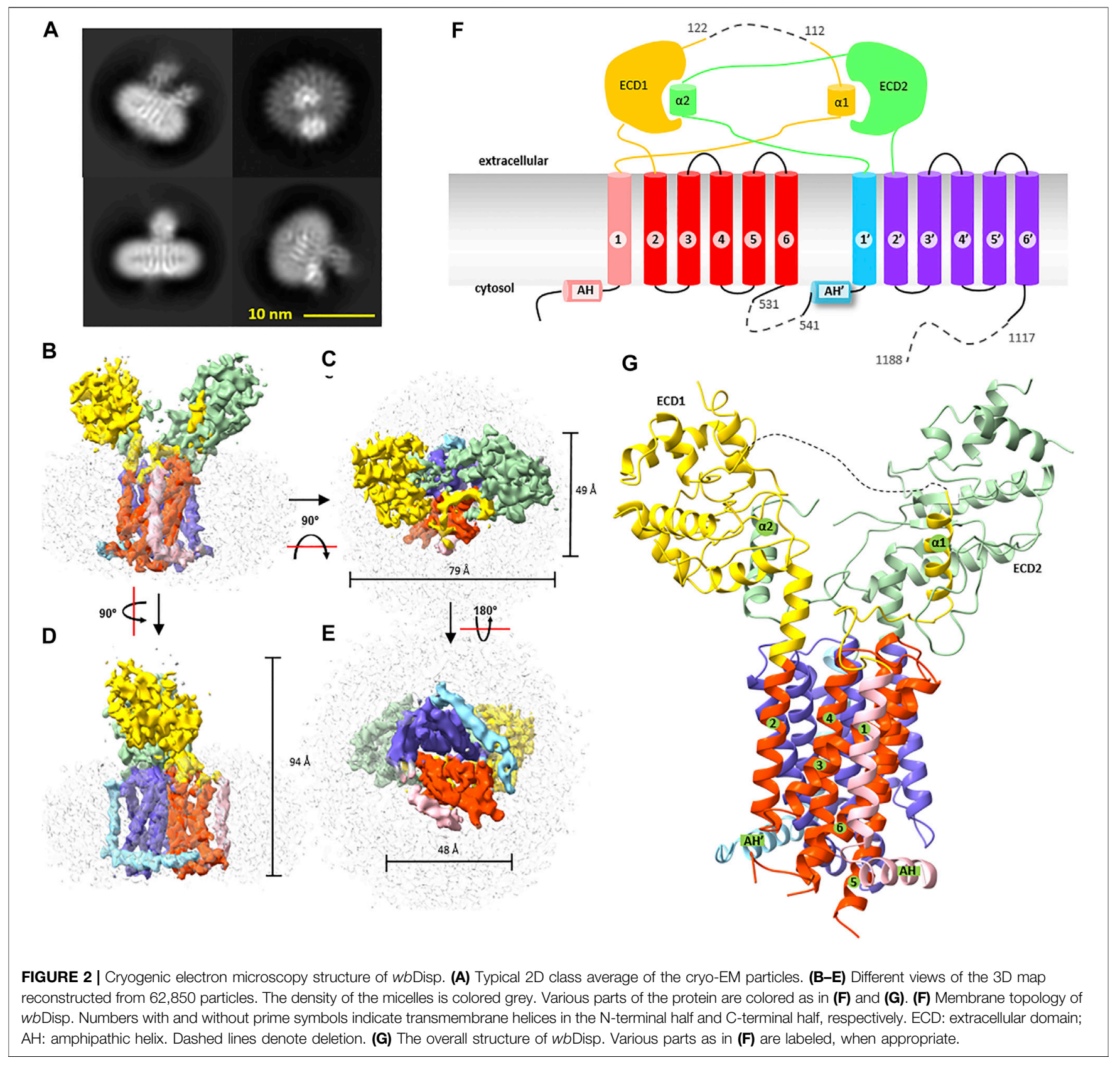

truncated at the C-terminal region, it is currently unknown if the wild type also exists as a trimer. However, since the wildtype $w b$ Disp had a similar retention volume as the truncated $w b \mathrm{Disp}^{*}$ (Figure 1F) on size exclusion chromatography, the protein engineering probably did not change the oligomerization state, at least under the micellar conditions. The possibility of Disp as oligomers remained to be investigated.

The $w b$ Disp structure has a dimension size of approximately 49, 79, and $94 \AA$. When viewed from the cytosol, the transmembrane region is square-shaped with a dimension of 49 by $48 \AA$ (Figures 2B-E). The two large extracellular domains (ECDs) occupy a similar volume of $\sim 3,350 \AA^{3}$.
The transmembrane domain shares a similar fold to other RND members. It contains two pseudosymmetrical repeats (Figure 2F, Supplementary Figure S9) with each repeat containing six TMHs and a soluble domain. Proceeding to the 6-TMH bundle is an amphipathic helix that lies on the membrane interface at the cytosol leaflet.

The two slingshot braches create a wide opening with a gap of $\sim 21 \AA$ at the bottom and of $\sim 32 \AA$ at the top (Figure 3A), enough to accommodate an $\mathrm{HhN}$ molecule which has a dimension of $\sim 20 \times 30 \times 38 \AA$ (Figure 3B). The opening is accessible from the vast extracellular space. Interestingly, when viewed perpendicular to the membrane, the exit to 

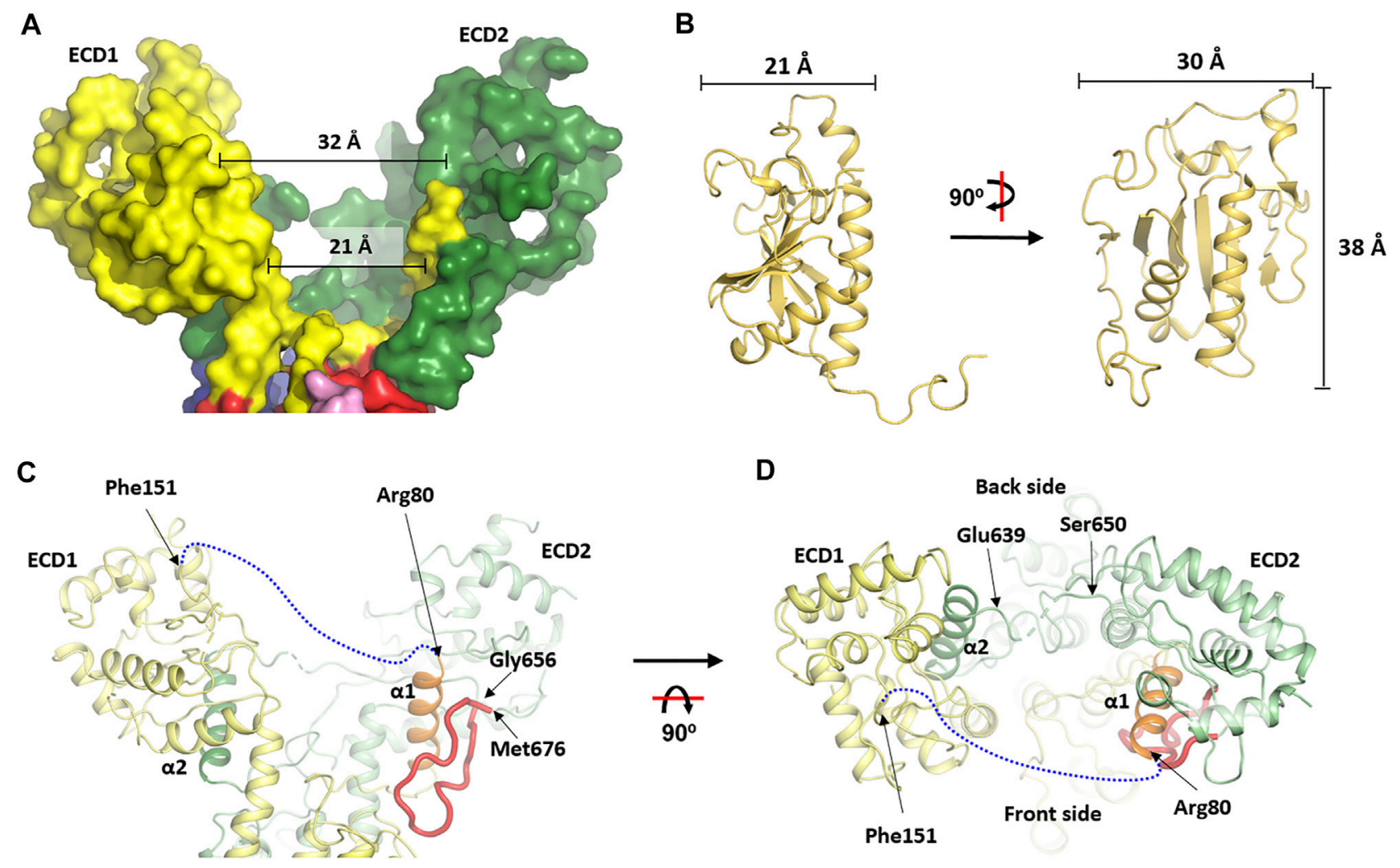

FIGURE 3 | The asymmetry of the extracellular domain and the conformational changes at the transmembrane domain. The two interconnected extracellular domains (ECDs) form the two arms of a slingshot and creates a cavity that is more open at the "front" side than at the "back" side. (A) surface view of the ECD part. (B) Dimension of the human HhN (PDB: 3M1N) (Pepinsky et al., 2000) which is close to the size of the cavity in (A). (C, D) Cartoon representation of the wbDisp structure at the two ECDs viewed from two different angles. Various parts are labeled when appropriate. A blue dash line indicates the ECD-connecting loop that was invisible in the current structure.

the "back" side (we name the site above TMH1-6 as the "front" side and the opposite as the "back" side) is somewhat restricted by a loop (res. 640-648) that connects the two branches (Figures 3C,D). Thus, embracing $\mathrm{HhN}$ in this direction would require large conformational rearrangements. By contrast, the loop (res. 80-159) at the "front" side is much longer and highly flexible (invisible in the structure), suggesting a more accommodating entrance for the presumable lateral diffusion of the membraneanchored $\mathrm{HhN}$.

One unique feature of the soluble domain is the way the two loops are interconnected. Thus, a small $\alpha$-helix ( $\alpha 1$, res. 66-79) stemming from TMH1 snakes to the slingshot branch made mostly by the ECD2 residues, before reaching back to form the other branch (ECD1) (Figures 3C,D). Reciprocally, the corresponding $\alpha$-helix in the C-half ( $\alpha 2$, res. 623-639) stemming from TMH7 also travels to the ECD1 part before making ECD2 (Figures 3C,D). Interestingly, a1 is clamped tightly onto the core ECD2 domain by a long loop while a2 only interacts with the core ECD1 at one side (Figures 3C,D). It might be that the binding and release of $\mathrm{Hh}$ is influenced by the formation and collapse of these unusual structural arrangements which may mediate cross-talk between the two ECDs.

\section{Structural Differences in the Transmembrane Domain and Possible Functional Consequences}

Recently, structures of the Disp from fruit fly (ffDisp) (Cannac et al., 2020) and human ( $h$ Disp) (Chen et al., 2020) have been reported. In addition to the apo form, the ffDisp structure was also determined in complex with the ligand $\mathrm{HhN}$. Interestingly, these published structures display little differences (Figure 4A). By contrast, noticeable differences, particularly at the transmembrane domain, were observed between the $w b$ Disp ${ }^{\star}$ and the published structures (Figures 4B-D). Specifically, the inner leaflet part of TMH1, TMH3, and TMH6 shift towards $\mathrm{TMH} 2$, compressing the membrane core region (Figure 4D), and this is repeated for the TMH1', TMH3', TMH6', and TMH2' in the C-terminal pseudosymmetrical half.

Although we acknowledge that the structural differences observed here should be treated with caution and should only be interpreted to a confident level the low-resolution map would 


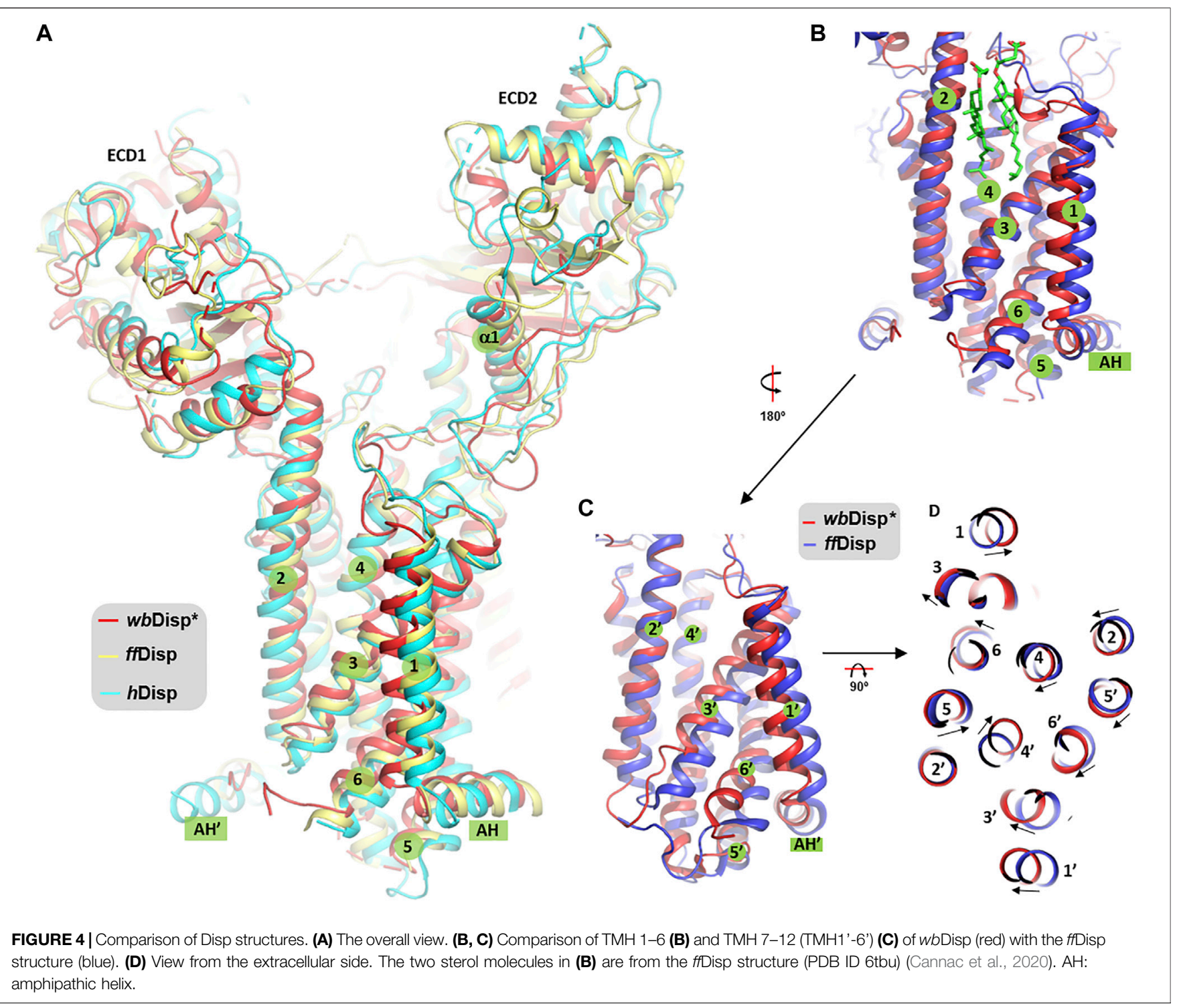

allow, the consequences of the structural shift are discussed below. The cleft between $\mathrm{TMH} 2$ and $\mathrm{TMH} 3$ is a sterol binding site, as revealed by the structure of ffDisp (Figure 4B); and this site, in the so-called sterol-sensing domain (SSD), is part of a sterol-transporting conduit for the homologous Ptc1 (Kowatsch et al., 2019) which consists of at least three sites (S1-S3, Supplementary Figure S10A). Additionally, in Ptcl, this conduit can be "plugged" by the N-terminal palmitoyl chain of $\mathrm{Hh}$ at the immediate downstream site S2 (Supplementary Figure S10B), that is, the Hh-binding activity and the steroltransport activity are structurally antagonistic. It is therefore possible that the conformational changes at this region are related to the sterol-transport function of $\mathrm{Ptc1}$, and by extension, the function of Disp assuming the two share a similar transporting mechanism. From the current limited resolution, it is not clear whether and how the conformational changes facilitate the binding and release of $\mathrm{Hh}$. Future structural studies in more native-like lipid environments at higher resolutions are warranted to test and amend this hypothesis.

\section{DATA AVAILABILITY STATEMENT}

The cryo-EM map has been deposited to EMDB with accession code EMD-31595 and the structure coordinates are available through the protein data bank (PDB) with entry ID 7FIF.

\section{ETHICS STATEMENT}

The animal study was reviewed and approved by the Institutional Animal Care and Use Committee, Shanghai Institute of Biochemistry and Cell Biology, Chinese Academy of Sciences. 


\section{AUTHOR CONTRIBUTIONS}

YL purified proteins with assistance from GW and QW, prepared EM samples, collected and processed EM data with advice from YC. GW screened constructs. XZ and YZhan performed functional assays under the supervision of YZhao. JB helped with cloning. DL and YZhao conceived the project. DL wrote the paper with inputs from YL and YZhao.

\section{FUNDING}

This work has been supported by the Strategic Priority Research Program of the Chinese Academy of Sciences (XDB37020204, XDB19020100), Key Program of CAS Frontier Science (QYZDBSSW-SMC037), CAS Facility-based Open Research Program, the National Natural Science Foundation of China (31870726, 31630047, 32130025), and the National Key Research and

\section{REFERENCES}

Bozzi, A. T., Bane, L. B., Weihofen, W. A., Singharoy, A., Guillen, E. R., Ploegh, H. L., et al. (2016). Crystal Structure and Conformational Change Mechanism of a Bacterial Nramp-Family Divalent Metal Transporter. Structure 24 (12), 2102-2114. doi:10.1016/j.str.2016.09.017

Burke, R., Nellen, D., Bellotto, M., Hafen, E., Senti, K.-A., Dickson, B. J., et al. (1999). Dispatched, a Novel Sterol-Sensing Domain Protein Dedicated to the Release of Cholesterol-Modified Hedgehog from Signaling Cells. Cell 99 (7), 803-815. doi:10.1016/s0092-8674(00)81677-3

Cai, H., Yao, H., Li, T., Hutter, C. A. J., Li, Y., Tang, Y., et al. (2020). An Improved Fluorescent Tag and its Nanobodies for Membrane Protein Expression, Stability Assay, and Purification. Commun. Biol. 3 (1), 753. doi:10.1038/ s42003-020-01478-z

Cannac, F., Qi, C., Falschlunger, J., Hausmann, G., Basler, K., and Korkhov, V. M. (2020). Cryo-EM Structure of the Hedgehog Release Protein Dispatched. Sci. Adv. 6 (16), eaay7928. doi:10.1126/sciadv.aay7928

Chang, Y., Bruni, R., Kloss, B., Assur, Z., Kloppmann, E., Rost, B., et al. (2014). Structural Basis for a $\mathrm{pH}$-Sensitive Calcium Leak across Membranes. Science 344 (6188), 1131-1135. doi:10.1126/science.1252043

Chen, H., Liu, Y., and Li, X. (2020). Structure of Human Dispatched-1 Provides Insights into Hedgehog Ligand Biogenesis. Life Sci. Alliance 3 (8). doi:10.26508/ lsa.202000776

Creanga, A., Glenn, T. D., Mann, R. K., Saunders, A. M., Talbot, W. S., and Beachy, P. A. (2012). Scube/You Activity Mediates Release of Dually Lipid-Modified Hedgehog Signal in Soluble Form. Genes Develop. 26 (12), 1312-1325. doi:10.1101/gad.191866.112

Doki, S., Kato, H. E., Solcan, N., Iwaki, M., Koyama, M., Hattori, M., et al. (2013). Structural Basis for Dynamic Mechanism of Proton-Coupled Symport by the Peptide Transporter POT. Proc. Natl. Acad. Sci. 110 (28), 11343-11348. doi:10.1073/pnas.1301079110

Emsley, P., Lohkamp, B., Scott, W. G., and Cowtan, K. (2010). Features and Development of Coot. Acta Crystallogr. D Biol. Crystallogr. 66 (Pt 4), 486-501. doi:10.1107/S0907444910007493

Etheridge, L. A., Crawford, T. Q., Zhang, S., and Roelink, H. (2010). Evidence for a Role of Vertebrate Disp1 in Long-Range Shh Signaling. Development 137 (1), 133-140. doi:10.1242/dev.043547

Eugster, C., Panáková, D., Mahmoud, A., and Eaton, S. (2007). Lipoproteinheparan Sulfate Interactions in the Hh Pathway. Develop. Cel 13 (1), 57-71. doi:10.1016/j.devcel.2007.04.019

Fukuda, Y., and Inoue, T. (2018). Crystal Structure of Secretory Abundant Heat Soluble Protein 4 from One of the Toughest "water Bears" Microanimals Ramazzottius Varieornatus. Protein Sci. 27 (5), 993-999. doi: $10.1002 /$ pro. 3393
Development Program of China (2020YFA0509000, 2017YFA0503600).

\section{ACKNOWLEDGMENTS}

We thank the staff members of the EM facility at the National Facility for Protein Science Shanghai and of the EM facility at ShanghaiTech University for assistance in data collection. We thank the staff members of the Large-Scale Protein Preparation System for equipment maintenance and management.

\section{SUPPLEMENTARY MATERIAL}

The Supplementary Material for this article can be found online at: https://www.frontiersin.org/articles/10.3389/fmolb.2021.701826/ full\#supplementary-material

Fukuda, Y., Miura, Y., Mizohata, E., and Inoue, T. (2017). Structural Insights into a Secretory Abundant Heat-Soluble Protein from an Anhydrobiotic Tardigrade, Ramazzottius Varieornatus. FEBS Lett. 591 (16), 2458-2469. doi:10.1002/18733468.12752

Gabriel, W. N., McNuff, R., Patel, S. K., Gregory, T. R., Jeck, W. R., Jones, C. D., et al. (2007). The Tardigrade Hypsibius Dujardini, a New Model for Studying the Evolution of Development. Develop. Biol. 312 (2), 545-559. doi:10.1016/ j.ydbio.2007.09.055

Galochkina, T., Ng Fuk ChongChong, M. M., Challali, L., Abbar, S., and Etchebest, C. (2019). New Insights into GluT1 Mechanics during Glucose Transfer. Sci. Rep. 9 (1), 998. doi:10.1038/s41598-018-37367-z

Gao, Y., Cao, E., Julius, D., and Cheng, Y. (2016). TRPV1 Structures in Nanodiscs Reveal Mechanisms of Ligand and Lipid Action. Nature 534 (7607), 347-351. doi:10.1038/nature17964

Goehring, A., Lee, C.-H., Wang, K. H., Michel, J. C., Claxton, D. P., Baconguis, I., et al. (2014). Screening and Large-Scale Expression of Membrane Proteins in Mammalian Cells for Structural Studies. Nat. Protoc. 9 (11), 2574-2585. doi:10.1038/nprot.2014.173

Goldstein, B. (2018). The Emergence of the Tardigrade Hypsibius Exemplaris as a Model System. Cold Spring Harb Protoc. 2018 (11). doi:10.1101/pdb.emo102301

Gong, X., Qian, H., Cao, P., Zhao, X., Zhou, Q., Lei, J., et al. (2018). Structural Basis for the Recognition of Sonic Hedgehog by Human Patched1. Science 361 (6402). doi:10.1126/science.aas 8935

Gong, X., Qian, H., Zhou, X., Wu, J., Wan, T., Cao, P., et al. (2016). Structural Insights into the Niemann-Pick C1 (NPC1)-Mediated Cholesterol Transfer and Ebola Infection. Cell 165 (6), 1467-1478. doi:10.1016/j.cell.2016.05.022

Hall, E. T., Dillard, M. E., Stewart, D. P., Zhang, Y., Wagner, B., Levine, R. M., et al. (2021). Cytoneme Delivery of Sonic Hedgehog from Ligand-Producing Cells Requires Myosin 10 and a Dispatched-BOC/CDON Co-receptor Complex. Elife 10, 10. doi:10.7554/eLife.61432

Hall, E. T., and Cleverdon, S. K. (2019). Dispatching Sonic Hedgehog: Molecular Mechanisms Controlling Deployment. Trends Cel Biol. 29 (5), 385-395. doi:10.1016/j.tcb.2019.02.005

Hattori, M., Hibbs, R. E., and Gouaux, E. (2012). A Fluorescence-Detection SizeExclusion Chromatography-Based Thermostability Assay for Membrane Protein Precrystallization Screening. Structure 20 (8), 1293-1299. doi:10.1016/j.str.2012.06.009

Hui, C.-c., and Angers, S. (2011). Gli Proteins in Development and Disease. Annu. Rev. Cel Dev. Biol. 27, 513-537. doi:10.1146/annurev-cellbio-092910-154048

Kalienkova, V., Clerico Mosina, V., Bryner, L., Oostergetel, G. T., Dutzler, R., and Paulino, C. (2019). Stepwise Activation Mechanism of the Scramblase nhTMEM16 Revealed by Cryo-EM. Elife 8. doi:10.7554/eLife.44364

Kim, J., Fukuda, Y., and Inoue, T. (2019). Crystal Structure of Kumaglobin: a Hexacoordinated Heme Protein from an Anhydrobiotic Tardigrade, Ramazzottius Varieornatus. FEBS J. 286 (7), 1287-1304. doi:10.1111/febs.14713 
Koleva, M. V., Rothery, S., Spitaler, M., Neil, M. A. A., and Magee, A. I. (2015). Sonic Hedgehog Multimerization: a Self-Organizing Event Driven by posttranslational Modifications? Mol. Membr. Biol. 32 (3), 65-74. doi:10.3109/ 09687688.2015.1066895

Kornberg, T. B. (2011). Barcoding Hedgehog for Intracellular Transport. Sci. Signaling 4 (200), pe44. doi:10.1126/scisignal.2002447

Kowatsch, C., Woolley, R. E., Kinnebrew, M., Rohatgi, R., and Siebold, C. (2019). Structures of Vertebrate Patched and Smoothened Reveal Intimate Links between Cholesterol and Hedgehog Signalling. Curr. Opin. Struct. Biol. 57, 204-214. doi:10.1016/j.sbi.2019.05.015

Kumar, S., Athreya, A., Gulati, A., Nair, R. M., Mahendran, I., Ranjan, R., et al. (2021). Structural Basis of Inhibition of a Transporter from Staphylococcus aureus, NorC, through a Single-Domain Camelid Antibody. Commun. Biol. 4 (1), 836. doi:10.1038/s42003-021-02357-x

Li, X., Wang, J., Coutavas, E., Shi, H., Hao, Q., and Blobel, G. (2016). Structure of Human Niemann-Pick C1 Protein. Proc. Natl. Acad. Sci. USA 113 (29), 8212-8217. doi:10.1073/pnas.1607795113

Liebschner, D., Afonine, P. V., Baker, M. L., Bunkóczi, G., Chen, V. B., Croll, T. I., et al. (2019). Macromolecular Structure Determination Using X-Rays, Neutrons and Electrons: Recent Developments in Phenix. Acta Crystallogr. D Struct. Biol. 75 (Pt 10), 861-877. doi:10.1107/S2059798319011471

Long, T., Qi, X., Hassan, A., Liang, Q., De Brabander, J. K., and Li, X. (2020). Structural Basis for Itraconazole-Mediated NPC1 Inhibition. Nat. Commun. 11 (1), 152. doi:10.1038/s41467-019-13917-5

Luo, Y., Wan, G., Zhang, X., Zhou, X., Wang, Q., Fan, J., et al. (2021). Cryo-EM Study of Patched in Lipid Nanodisc Suggests a Structural Basis for its Clustering in Caveolae. Structure. doi:10.1016/j.str.2021.06.004

Ma, Y., Erkner, A., Gong, R., Yao, S., Taipale, J., Basler, K., et al. (2002). Hedgehogmediated Patterning of the Mammalian Embryo Requires Transporter-like Function of Dispatched. Cell 111 (1), 63-75. doi:10.1016/s0092-8674(02)00977-7

Mao, H., Diehl, A. M., and Li, Y.-X. (2009). Sonic Hedgehog Ligand Partners with Caveolin-1 for Intracellular Transport. Lab. Invest. 89 (3), 290-300. doi:10.1038/labinvest.2008.163

Mastronarde, D. N. (2005). Automated Electron Microscope Tomography Using Robust Prediction of Specimen Movements. J. Struct. Biol. 152 (1), 36-51. doi:10.1016/j.jsb.2005.07.007

Matthies, D., Bae, C., Toombes, G. E., Fox, T., Bartesaghi, A., Subramaniam, S., et al. (2018). Single-particle Cryo-EM Structure of a Voltage-Activated Potassium Channel in Lipid Nanodiscs. Elife 7. doi:10.7554/eLife.37558

McGuffin, L. J., Bryson, K., and Jones, D. T. (2000). The PSIPRED Protein Structure Prediction Server. Bioinformatics 16 (4), 404-405. doi:10.1093/ bioinformatics/16.4.404

Mi, W., Li, Y., Yoon, S. H., Ernst, R. K., Walz, T., and Liao, M. (2017). Structural Basis of MsbA-Mediated Lipopolysaccharide Transport. Nature 549 (7671), 233-237. doi:10.1038/nature23649

Mindell, J. A., and Grigorieff, N. (2003). Accurate Determination of Local Defocus and Specimen Tilt in Electron Microscopy. J. Struct. Biol. 142 (3), 334-347. doi:10.1016/s1047-8477(03)00069-8

Myers, B. R., Neahring, L., Zhang, Y., Roberts, K. J., and Beachy, P. A. (2017). Rapid, Direct Activity Assays for Smoothened Reveal Hedgehog Pathway Regulation by Membrane Cholesterol and Extracellular Sodium. Proc. Natl. Acad. Sci. USA 114 (52), E11141-E11150. doi:10.1073/pnas.1717891115

Newstead, S., Drew, D., Cameron, A. D., Postis, V. L. G., Xia, X., Fowler, P. W., et al. (2011). Crystal Structure of a Prokaryotic Homologue of the Mammalian Oligopeptide-Proton Symporters, PepT1 and PepT2. EMBO J. 30 (2), 417-426. doi:10.1038/emboj.2010.309

Nieuwenhuis, E., and Hui, C. C. (2005). Hedgehog Signaling and Congenital Malformations. Clin. Genet. 67 (3), 193-208. doi:10.1111/j.13990004.2004.00360.x

Niewiadomski, P., Niedziółka, S. M., Markiewicz, Ł., Uśpieński, T., Baran, B., and Chojnowska, K. (2019). Gli Proteins: Regulation in Development and Cancer. Cells 8 (2). doi:10.3390/cells8020147

Nikaido, H. (2018). RND Transporters in the Living World. Res. Microbiol. 169 (78), 363-371. doi:10.1016/j.resmic.2018.03.001

Nüsslein-Volhard, C., and Wieschaus, E. (1980). Mutations Affecting Segment Number and Polarity in Drosophila. Nature 287 (5785), 795-801. doi:10.1038/ $287795 \mathrm{a} 0$
Orellana, R., Macaya, C., Bravo, G., Dorochesi, F., Cumsille, A., Valencia, R., et al. (2018). Living at the Frontiers of Life: Extremophiles in Chile and Their Potential for Bioremediation. Front. Microbiol. 9, 2309. doi:10.3389/ fmicb.2018.02309

Otsuka, A., Levesque, M. P., Dummer, R., and Kabashima, K. (2015). Hedgehog Signaling in Basal Cell Carcinoma. J. Dermatol. Sci. 78 (2), 95-100. doi:10.1016/ j.jdermsci.2015.02.007

Panáková, D., Sprong, H., Marois, E., Thiele, C., and Eaton, S. (2005). Lipoprotein Particles Are Required for Hedgehog and Wingless Signalling. Nature 435 (7038), 58-65. doi:10.1038/nature03504

Pepinsky, R. B., Rayhorn, P., Day, E. S., Dergay, A., Williams, K. P., Galdes, A., et al. (2000). Mapping Sonic Hedgehog-Receptor Interactions by Steric Interference. J. Biol. Chem. 275 (15), 10995-11001. doi:10.1074/jbc.275.15.10995

Pepinsky, R. B., Zeng, C., Wen, D., Rayhorn, P., Baker, D. P., Williams, K. P., et al. (1998). Identification of a Palmitic Acid-Modified Form of Human Sonic Hedgehog. J. Biol. Chem. 273 (22), 14037-14045. doi:10.1074/jbc.273.22.14037

Pettersen, E. F., Goddard, T. D., Huang, C. C., Couch, G. S., Greenblatt, D. M., Meng, E. C., et al. (2004). UCSF Chimera?A Visualization System for Exploratory Research and Analysis. J. Comput. Chem. 25 (13), 1605-1612. doi:10.1002/jcc.20084

Porter, J. A., Ekker, S. C., Park, W.-J., von Kessler, D. P., Young, K. E., Chen, C.-H., et al. (1996). Hedgehog Patterning Activity: Role of a Lipophilic Modification Mediated by the Carboxy-Terminal Autoprocessing Domain. Cell 86 (1), 21-34. doi:10.1016/s0092-8674(00)80074-4

Porter, J. A., Young, K. E., and Beachy, P. A. (1996). Cholesterol Modification of Hedgehog Signaling Proteins in Animal Development. Science 274 (5285), 255-259. doi:10.1126/science.274.5285.255

Pos, K. M. (2009). Drug Transport Mechanism of the AcrB Efflux Pump. Biochim. Biophys. Acta (Bba) - Proteins Proteomics 1794 (5), 782-793. doi:10.1016/ j.bbapap.2008.12.015

Qi, C., Di Minin, G., Vercellino, I., Wutz, A., and Korkhov, V. M. (2019). Structural Basis of Sterol Recognition by Human Hedgehog Receptor PTCH1. Sci. Adv. 5 (9), eaaw6490. doi:10.1126/sciadv.aaw6490

Qi, X., Schmiege, P., Coutavas, E., and Li, X. (2018). Two Patched Molecules Engage Distinct Sites on Hedgehog Yielding a Signaling-Competent Complex. Science 362 (6410). doi:10.1126/science.aas8843

Qi, X., Schmiege, P., Coutavas, E., Wang, J., and Li, X. (2018). Structures of Human Patched and its Complex with Native Palmitoylated Sonic Hedgehog. Nature 560 (7716), 128-132. doi:10.1038/s41586-018-0308-7

Qian, H., Cao, P., Hu, M., Gao, S., Yan, N., and Gong, X. (2019). Inhibition of Tetrameric Patched1 by Sonic Hedgehog through an Asymmetric Paradigm. Nat. Commun. 10 (1), 2320. doi:10.1038/s41467-019-10234-9

Qian, H., Wu, X., Du, X., Yao, X., Zhao, X., Lee, J., et al. (2020). Structural Basis of Low-pH-dependent Lysosomal Cholesterol Egress by NPC1 and NPC2. Cell 182 (1), 98-111. doi:10.1016/j.cell.2020.05.020

Reddy, B., Bavi, N., Lu, A., Park, Y., and Perozo, E. (2019). Molecular Basis of Force-From-Lipids Gating in the Mechanosensitive Channel MscS. Elife 8. doi:10.7554/eLife.50486

Rudolf, A. F., Kinnebrew, M., Kowatsch, C., Ansell, T. B., El Omari, K., Bishop, B., et al. (2019). The Morphogen Sonic Hedgehog Inhibits its Receptor Patched by a Pincer Grasp Mechanism. Nat. Chem. Biol. 15 (10), 975-982. doi:10.1038/ s41589-019-0370-y

Scheres, S. H. W. (2012). A Bayesian View on Cryo-EM Structure Determination. J. Mol. Biol. 415 (2), 406-418. doi:10.1016/j.jmb.2011.11.010

Scheres, S. H. W. (2012). RELION: Implementation of a Bayesian Approach to Cryo-EM Structure Determination. J. Struct. Biol. 180 (3), 519-530. doi:10.1016/j.jsb.2012.09.006

Seeger, M. A., Schiefner, A., Eicher, T., Verrey, F., Diederichs, K., and Pos, K. M. (2006). Structural Asymmetry of AcrB Trimer Suggests a Peristaltic Pump Mechanism. Science 313 (5791), 1295-1298. doi:10.1126/science.1131542

Simon, E., Aguirre-Tamaral, A., Aguilar, G., and Guerrero, I. (2016). Perspectives on Intra- and Intercellular Trafficking of Hedgehog for Tissue Patterning. J. Dev. Biol. 4 (4). doi:10.3390/jdb4040034

Sloan, D., Alves Batista, R., and Loeb, A. (2017). The Resilience of Life to Astrophysical Events. Sci. Rep. 7 (1), 5419. doi:10.1038/s41598-017-05796-x

Solcan, N., Kwok, J., Fowler, P. W., Cameron, A. D., Drew, D., Iwata, S., et al. (2012). Alternating Access Mechanism in the POT Family of Oligopeptide Transporters. EMBO J. 31 (16), 3411-3421. doi:10.1038/emboj.2012.157 
Taipale, J., Cooper, M. K., Maiti, T., and Beachy, P. A. (2002). Patched Acts Catalytically to Suppress the Activity of Smoothened. Nature 418 (6900), 892-896. doi:10.1038/nature00989

Tsiairis, C. D., and McMahon, A. P. (2008). Disp1 Regulates Growth of Mammalian Long Bones through the Control of Ihh Distribution. Develop. Biol. 317 (2), 480-485. doi:10.1016/j.ydbio.2008.02.039

Tukachinsky, H., Kuzmickas, R. P., Jao, C. Y., Liu, J., and Salic, A. (2012). Dispatched and Scube Mediate the Efficient Secretion of the Cholesterol-Modified Hedgehog Ligand. Cel Rep. 2 (2), 308-320. doi:10.1016/j.celrep.2012.07.010

Varshney, G. K., Pei, W., LaFave, M. C., Idol, J., Xu, L., Gallardo, V., et al. (2015). High-throughput Gene Targeting and Phenotyping in Zebrafish Using CRISPR/Cas9. Genome Res. 25 (7), 1030-1042. doi:10.1101/gr.186379.114

Vuppada, R. K., Hansen, C. R., Strickland, K. A. P., Kelly, K. M., and McCleary, W. R. (2018). Phosphate Signaling through Alternate Conformations of the PstSCAB Phosphate Transporter. BMC Microbiol. 18 (1), 8. doi:10.1186/s12866-017-1126-Z

Wulhfard, S., Baldi, L., Hacker, D. L., and Wurm, F. (2010). Valproic Acid Enhances Recombinant mRNA and Protein Levels in Transiently Transfected Chinese Hamster Ovary Cells. J. Biotechnol. 148 (2-3), 128-132. doi:10.1016/j.jbiotec.2010.05.003

Yao, H., Cai, H., and Li, D. (2020). Thermostabilization of Membrane Proteins by Consensus Mutation: A Case Study for a Fungal $\Delta 8-7$ Sterol Isomerase. J. Mol. Biol. 432 (18), 5162-5183. doi:10.1016/j.jmb.2020.02.015

Zhang, Y., Bulkley, D. P., Xin, Y., Roberts, K. J., Asarnow, D. E., Sharma, A., et al. (2018). Structural Basis for Cholesterol Transport-like Activity of the Hedgehog Receptor Patched. Cell 175 (5), 1352-1364. doi:10.1016/j.cell.2018.10.026
Zhang, Y., Lu, W.-J., Bulkley, D. P., Liang, J., Ralko, A., Han, S., et al. (2020) Hedgehog Pathway Activation through Nanobody-Mediated Conformational Blockade of the Patched Sterol Conduit. Proc. Natl. Acad. Sci. USA 117 (46), 28838-28846. doi:10.1073/pnas.2011560117

Zimmermann, I., Egloff, P., Hutter, C. A., Arnold, F. M., Stohler, P., Bocquet, N., et al. (2018). Synthetic Single Domain Antibodies for the Conformational Trapping of Membrane Proteins. Elife 7. doi:10.7554/eLife.34317

Conflict of Interest: The authors declare that the research was conducted in the absence of any commercial or financial relationships that could be construed as a potential conflict of interest.

Publisher's Note: All claims expressed in this article are solely those of the authors and do not necessarily represent those of their affiliated organizations, or those of the publisher, the editors, and the reviewers. Any product that may be evaluated in this article, or claim that may be made by its manufacturer, is not guaranteed or endorsed by the publisher.

Copyright (c) 2021 Luo, Wan, Zhou, Wang, Zhang, Bao, Cong, Zhao and Li. This is an open-access article distributed under the terms of the Creative Commons Attribution License (CC BY). The use, distribution or reproduction in other forums is permitted, provided the original author(s) and the copyright owner(s) are credited and that the original publication in this journal is cited, in accordance with accepted academic practice. No use, distribution or reproduction is permitted which does not comply with these terms. 